\title{
The interplay between Peierls distortions and metavalent bonding in IV-VI compounds: comparing GeTe with related monochalcogenides
}

\author{
Jean-Yves Raty ${ }^{1,2}$ and Matthias Wuttig ${ }^{3}$ \\ ${ }^{1}$ FNRS, SPIN-CESAM, Université de Liège, 19 Allée du 6Août, 4000 Sart-Tilman, Belgium \\ ${ }^{3}$ Univ. Grenoble Alpes, CEA, LETI, F-38000 Grenoble, France \\ ${ }^{3}$ Institute of Physics IA, RWTH Aachen University, 52074 Aachen, Germany \\ and JARA-Institute: Energy-Efficient Information Technology (Green IT), \\ Forschungszentrum Jülich GmbH, 52428 Jülich, Germany \\ E-mail: jyraty@ulg.ac.be
}

Received xxxxxx

Accepted for publication $\mathrm{xxxxxx}$

Published xxxxxx

\begin{abstract}
In this article, we revisit bonding in crystalline $\mathrm{GeTe}$, a simple binary alloy that is also a popular Phase Change Material, and use an ab initio approach that goes beyond the usual one electron description obtained with Density Functional Theory. By considering the electron pair density, we obtain a measure of the number of pairs of electrons that are shared between neighbors. Employing the charge transfer between adjacent atoms as the second quantifier of chemical bonding, we obtain a map which separates ionic, covalent and metallic bonding. Interestingly, GeTe is not located in any of these regions, but instead is located in a region where materials with a peculiar set of properties prevails. The corresponding materials have been coined incipient metals and their bonding 'metavalent bonding' (MVB). They often possess a Peierls distortion, which stabilizes the rhombohedral crystal structure by breaking the cubic symmetry. For these materials, the electron population of longer and shorter bonds is close to one-half, and charge transfer between adjacent atoms is quasi-independent of the degree of distortion. The energy gained by the Peierls distortion is much smaller than the energy gained by creating the cubic structure, delocalizing one electron over two bonds. Such Peierls distortions are not observed for aromatic compounds which utilize resonant bonding and have properties which differ significantly from the property portfolio of metavalently bonded materials. This stresses the difference between metavalent bonding and the resonant valence bond view of aromatic compounds and molecules. MVB is also responsible for the anomalies in dielectric properties and the anharmonicity of the solids. The comparison between PbTe, GeTe and GeS is particularly instructive, showing that bonding in these materials shows interesting differences, where metavalent bonds govern the behavior of PbTe and GeTe, while GeS is dominated by the Peierls distortion.
\end{abstract}

Keywords: Phase Change Material, Metavalent Bonding, Ab Initio simulation

\section{Introduction}

Almost 50 years ago, Lucovsky and White published a remarkable paper which discussed and explained pronounced 
property differences between amorphous and crystalline IV VI semiconductors like GeTe and GeSe [1]. These dissimilarities are surprising, since ordinary covalent network formers like $\mathrm{SiO} 2$ show hardly any property difference between the amorphous and crystalline state; a finding that was already explained in 1932 by Zachariasen [2]. He argued that oxide glasses show the same bonding as the corresponding crystal and hence the same short range order. Lucovsky and White, instead, explained the pronounced difference in atomic arrangement between amorphous and crystalline GeTe (and GeSe) by a difference in bonding mechanism for the two different phases. While the amorphous phase utilizes covalent bonding, according to Lucovsky and White the crystalline phase employs resonance bonding. This difference in bonding helps to explain the pronounced property contrast between both phases. 35 years ago, this property contrast was exploited for reversible (re-writeable) optical data storage employing GeTe. Just two years later, the first commercial product was introduced, which utilized a rewriteable optical disk [3] and employed phase change materials on the pseudo-binary line between GeTe and $\mathrm{Sb} 2 \mathrm{Te} 3$, such as Ge2Sb2Te5. Subsequently, further applications of related chalcogenides have been developed and introduced to the market such as the Optane Memory, jointly developed by Intel and Micron [4]; a non-volatile electronic memory which utilizes similar chalcogenides. One of the most important aspects of PCMs for this technology resides is the fact that their liquid phase is highly fragile and their amorphous phase can recrystallize in extremely short times (nanosecond timescale [5]). Understanding the relation between bonding in the amorphous and in the crystalline phase appears to be a key factor to understand the crystallisation kinetics [6-8]. In recent years, other applications, among which numerous photonic applications of phase change materials have been suggested including displays, metasurfaces [9-11], electro-optic devices or photonic memories [12].

In many of these publications, the remarkable property portfolio of crystalline chalcogenides is attributed to resonant (or resonance) bonding. Yet, the idea of resonant bonding has been questioned in recent papers [13-15]. While these papers acknowledge and often reproduce pronounced differences in properties between the amorphous and crystalline state, they argue that bonding in crystalline phase change materials differs from resonance/resonant bonding. For example, Mukhopadhyay et al. calculated a pronounced difference of the Born effective charge between the amorphous and crystalline phase of Ge2Sb2Te5 [16]. The Born effective charge characterizes the dynamic dipole moment, i.e. the dipole moment created upon a vibronic displacement pattern and is thus a measure of the chemical bond polarizability. Indeed, the pronounced difference in the chemical bond polarizability had already been found earlier in experiments and simulations studying the properties of the amorphous and crystalline state of phase change materials such as GeTe [17]. A pronounced difference of the Born effective charges of amorphous and crystalline Ge2Sb2Te5 has also recently been reported by Lee and Elliott, but is attributed to covalent bonding, instead [13]. More precisely, they argue that amorphous Ge2Sb2Te5 utilizes ordinary covalent bonding, while crystalline Ge2Sb2Te5 employs 'hypervalent' bonding. Yet, the manuscript does not provide a unique definition of the property portfolio that characterizes 'hypervalent' bonding. Hence all recent studies which investigate the Born effective charges in amorphous and crystalline phase change materials agree that those differ significantly between the amorphous and the crystalline state, but provide conflicting explanations for this finding. Interestingly, the story becomes even more puzzling, when one looks at related IV-VI compounds from the perspective of the solid state physicist [18, 19], who stresses the similarity of compounds such as PbTe (a wellknown thermoelectric) and GeTe (the first phase change material). Yet, PbTe possesses an undistorted rocksalt structure, frequently attributed to ionic bonding, while GeTe features a distorted rocksalt structure, which is frequently ascribed to an electronic instability leading to a Peierls distortion [18-21]

Indeed, the periodic alternation of shorter and longer bonds opens a gap at the Fermi level which is located in the middle of the half-filled density of p-states. The Peierls distortion lowers the energy of the highest occupied states, stabilizing the structure. The atomic arrangement is either rhombohedral as in GeTe, or orthorhombic as in $\mathrm{GeS}, \mathrm{GeSe}, \mathrm{SnS}$, if the elastic energy cost due to the repulsive interaction between ionic cores remains small $[22,23]$. The heaviest compounds have steeper effective repulsive potentials between cores which prevent any distortion, and are therefore cubic (PbSe, $\mathrm{PbTe})$. This view of the chemical bonding in IV-VI chalcogenides has been widely used to account for many phenomena observed in these compounds, for instance to account for the temperature and pressure induced phase transitions (the distorted systems become cubic [24, 25]) or for the characteristics of the liquid and amorphous phases [17, 2629]. However, we are not aware of any theory based on the Peierls distortion or covalent bonding, which can predict for which chalcogenides a pronounced difference exists between the amorphous and crystalline state. The lack of such a theory based on the Peierls distortion and/or covalent bonding indicates that claims of the existence of design strategies for phase change materials still might have to be taken with caution.

The first attempt to explain the difference between amorphous and crystalline GeTe (as well as GeSe), was the suggestion by Lucovsky and White, to stress the analogy with aromatic molecules and graphite (or graphene) in which delocalization of electrons occurs from resonating $\pi$ states. In 
the resonant valence bond picture, Lucovsky and White established a model for IV-VI compounds in which Peierls distorted states with different distortion are combined to create a resonant state of lower energy [1]. They argued that this can explain the pronounced contrast of the optical properties between the amorphous and crystalline state of GeTe. Indeed, almost 40 years later it was shown that many phase change materials possess a pronounced difference of their optical properties such as $\varepsilon_{\infty}$ [30], but also their vibrational properties [31] and their atomic arrangement [32]. Subsequently, a first map was suggested to locate suitable phase change materials based on the concept of resonant bonding [33]. This resonant bonding description has become increasingly popular, as it could easily explain the optical contrast between amorphous (disordered network of mostly pbonded atoms) and crystalline phases of Phase Change Materials (which includes GeTe) [30, 34]. Recently, resonant bonding in $\mathrm{Pb}$ and $\mathrm{Sn}$ tellurides has been correlated with the enhanced thermoelectric figure of merit in these compounds. Actually, those cubic compounds described as resonantly bonded exhibit soft vibrational modes which decrease the thermal conductivity [35]. The fact that the excellent thermoelectric properties of PbTe and the $\alpha$-phase of SnSe have been frequently ascribed to lone pairs adds further potential for confusion regarding the bonding origin of the structure - property relationship. Quite surprisingly, lone pairs have also been presented as a key feature of bonding in amorphous [36] and crystalline GeTe [37], although it had been shown earlier by Waghmare et al. that lone pairs were very much incomplete due to a poor mixing of $\mathrm{s}$ and $\mathrm{p}$ states on the cation [38].

In an attempt to tackle this confusion, we revisit the bonding in GeTe and compare it to two other IV-VI compounds, i.e. PbTe and GeS.

Very recently, IV-VI compounds were compared to more than one hundred crystal compositions using a quantum chemically based method that is able to compute the number of electrons shared or transferred between atoms. This allowed to quantify bonding (locate solids in unique regions which are dominated by ionic, metallic and covalent bonding) and establish the relation between bonding and the anomalous (dielectric, vibrational) properties that are observed in some IV-VI $[15,39,40]$ and related compounds such as V-VI ones (Sb2Te3, for instance [40]). It was shown that all the anomalous properties are observed when the number of electrons effectively shared in bonds is close to one. The compounds exhibiting such a particular bond half-filling are clearly distinct from well-known covalent, ionic and metallic compounds. Therefore, these unconventional compounds have been called metavalent (for Metavalently Bonded Compounds, or MVB). At the same time, it was shown that simply extending the Resonant Valence Bond (RVB) picture from aromatic systems to those chalcogenides compounds and related solids in the resonant bonding picture could not be justified for several reasons. First the anomalous properties observed in MVB compounds are not observed in aromatic systems (including graphite/graphene), which can be explained by the fact that in MVB compounds, the same electrons are responsible for bonding and for the response properties (vibrational, optical, electronic), whereas in the RVB compounds, bonding and vibrational properties are essentially insured by the s-p backbone whereas transport is driven by the physics of resonating $\pi$ states.

Recently, strong further experimental evidence for the uniqueness of metavalent bonding has been provided by Atom Probe tomography [41]. Without any exception, all MVB crystals studied in the atom probe showed a very unconventional bond rupture. In laser-assisted field evaporation usually the probability to from more than one fragment is very low, i.e. around $10-20 \%$. However, in solids which employ MVB, this probability to form more than one fragment was more than $60 \%$. Such a bond rupture was not observed for carbon nanotubes, which utilize resonant bonding [42]. This confirms that indeed resonant bonding and metavalent bonding are different bonding mechanisms.

In this paper, we present an ab initio study of selected IVVI compounds to describe metavalent bonding in detail, by analyzing the interplay between delocalization (as in metallic bonding) and localization (as in covalent bonding as well as ionic bonding). We show that MVB provides a coherent picture of bonding and properties with has predictive power, while neither ionic bonding nor lone pairs play a major role in the case of PbTe, GeTe and GeS.

\section{Methods}

The ab Initio calculations were performed in the framework of Density Functional Theory (DFT). Three different plane wave basis codes were employed, including ABINIT [43], VASP [44, 45] and PWSCF [46]. The VASP code was used in conjunction with PAW potentials [44] and PBE exchange correlation, while ABINIT was utilized with norm-conserving potentials and PBE [47], PWSCF was applied with normconserving [48] and PAW potentials together with PBE [49] and PBESOL [50] exchange correlation functionals. The PBESOL functional has been shown to provide improved structural parameters for layered $\mathrm{Ge}_{\mathrm{x}} \mathrm{Sb}_{\mathrm{y}} \mathrm{Te}_{\mathrm{z}}$ crystals [51] and was used in the calculation of the reduced kinetic energy density and Wannier functions. The linear response features were computed as described in Ref. [52].

The structures have been relaxed to less than $1 \mathrm{E}-5 \mathrm{eV} / \mathrm{A}$ to compute the phonon dispersion curves and the plane wave cutoff was chosen large enough to ensure convergence of the energy to less than $1 \mathrm{meV} /$ atom. The initial DFT wavefunctions have then been post-processed either in the DGRID code [53] or followed by a transformation into 
maximally localized Wannier functions $[54,55]$ and integration [56] within the Critic2 code [57] to obtain the localization and delocalization indices for individual atoms. The PBE exchange correlation functional was used for these calculations in order to obtain results that are directly comparable with previously publications $[15,39,40]$.

\section{Results and Discussion}

The relevant structures for IV-VI compounds are illustrated in Figure 1 for GeTe. The stable phase (R-3m) arises from the Peierls distortion in the $\langle 111\rangle$ direction of the rocksalt structure, which is that of the stable phase of PbTe and PbSe. As a consequence of the distortion, the first shell of neighbors is divided into two subshells, with shorter bonds (2.86 $⿱$ A here) facing longer ones (3.21 $\AA$ ). The Peierls distortion has a very limited effect on the bonding angle since the angle between short bonds is $96^{\circ}$, as compared to $90^{\circ}$ for the perfect cubic structure and that between almost aligned (short-long) bonds equals $171^{\circ}$, as compared to $180^{\circ}$ for the perfect reference. However, the distortion is sufficient to increase the energy gap by lowering the energy of the uppermost valence states (see Figure 2), the gain in energy reaching $0.013 \mathrm{eV}$ in the case of GeTe (PBE calculations). Ref. [58] gives a detailed account of the structural, electronic and dielectric properties of the phases.
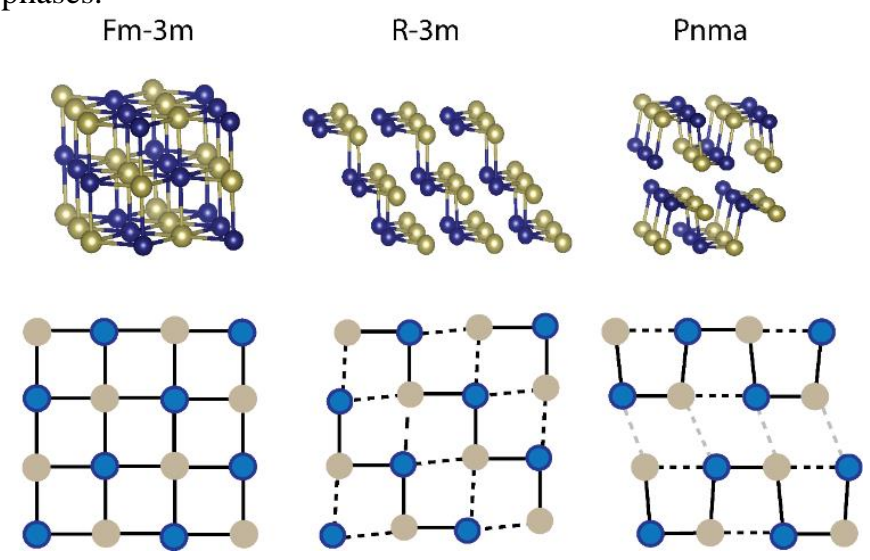

Figure 1: Relationship between cubic (Fm-3m, left), rhombohedral (R-3m, center) and orthorhombic (Pnma, right) phases of IV-VI compounds. The 2D schematic representations show how the various structures derive from the cubic one (rocksalt structure) by creating distortions (R-3m, Pnma) and impose a translation to bilayers of atoms (Pnma).

Alternatively, the distortion from the symmetric cubic structure can be seen as a ferroelectric instability due to an unstable phonon at the $\Gamma$ point in the cubic phase [59], however the high concentration of intrinsic defects (hole carriers) prevents GeTe from being a true ferroelectric [60], i.e. developing a macroscopic polarization in the bulk

The last structure that is relevant for IV-VI chalcogenides is the orthorhombic (Pnma or its enantiomorph Pbnm) structure, which is the stable structure of GeSe and GeS, for instance. This structure is also obtained from a Peierls distortion of the rocksalt structure and has been commonly described as the stacking of covalently bonded bilayers due to van der Waals forces. Figure 1 shows that one can draw a
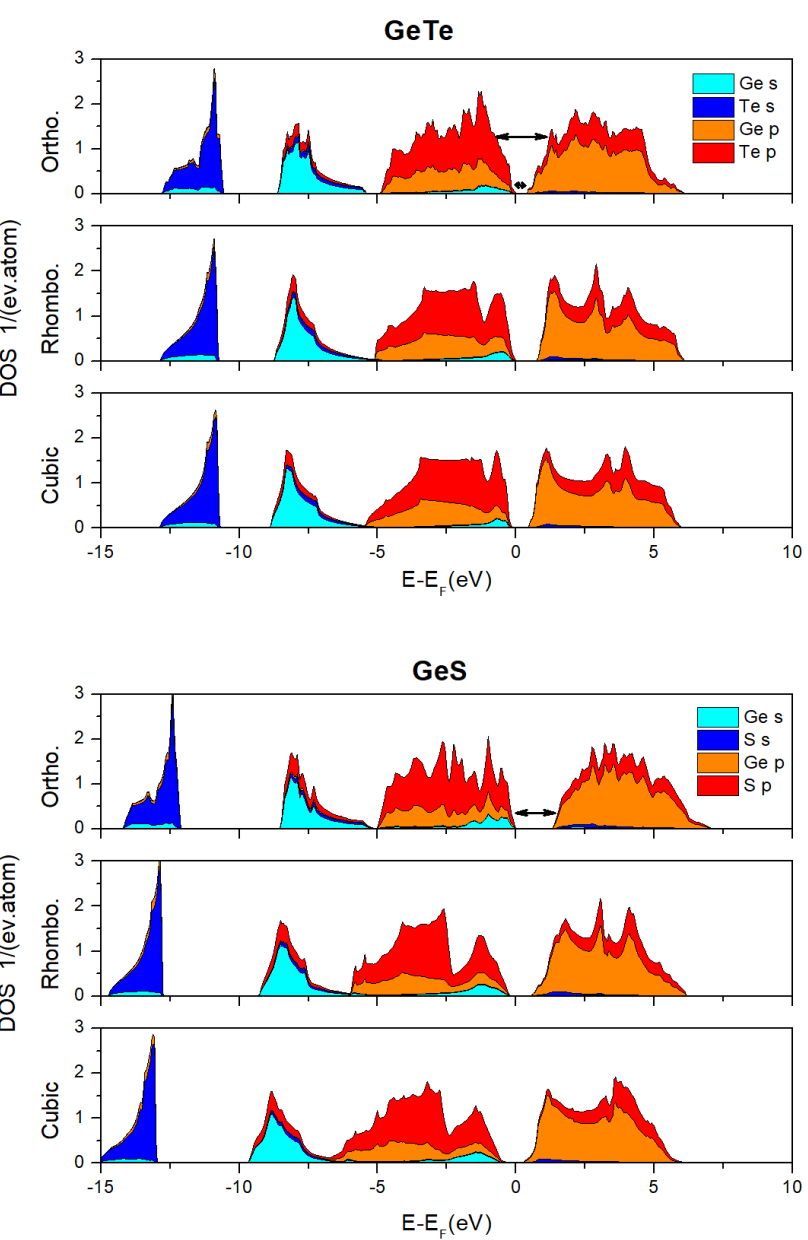

Figure 2: Density of states for various crystalline structures of GeTe (top three panels) and GeS (bottom three panels), computed with the GGA PBE. For each system, from bottom to top: rocksalt structure, R-3m structure (the Peierls distortion ratio of the equilibrium GeTe structure is used in both systems, with all other parameters relaxed) and orthorhombic Pnma structure (equilibrium GeS structure, constrained relaxation for GeTe). The total DOS is decomposed as the sum of partial densities (stacked curves here) analyzed with the Lobster code [61]. 
continuous transformation between rocksalt, rhombohedral and orthorhombic structures.

In the case of $\mathrm{GeS}$, the energy gained by distorting the rocksalt structure $(0.048 \mathrm{eV}$, this work) is much larger than in GeTe. The amplitude of the Peierls distortion is also larger, ranging between 1.37 in the bilayers and 1.52 perpendicular to gap in the stable $\mathrm{R}-3 \mathrm{~m}$ phase is larger than that in the (unstable) orthorhombic phase. In the Pnma phase one has to distinguish between the in-plane and the out-of-plane directions. Out of plane, the initial Peierls distortion is followed by a translation which breaks the alignment of 'shorter' and 'longer' bonds (see Figure 1). This would

\section{Electron Localisation Function}
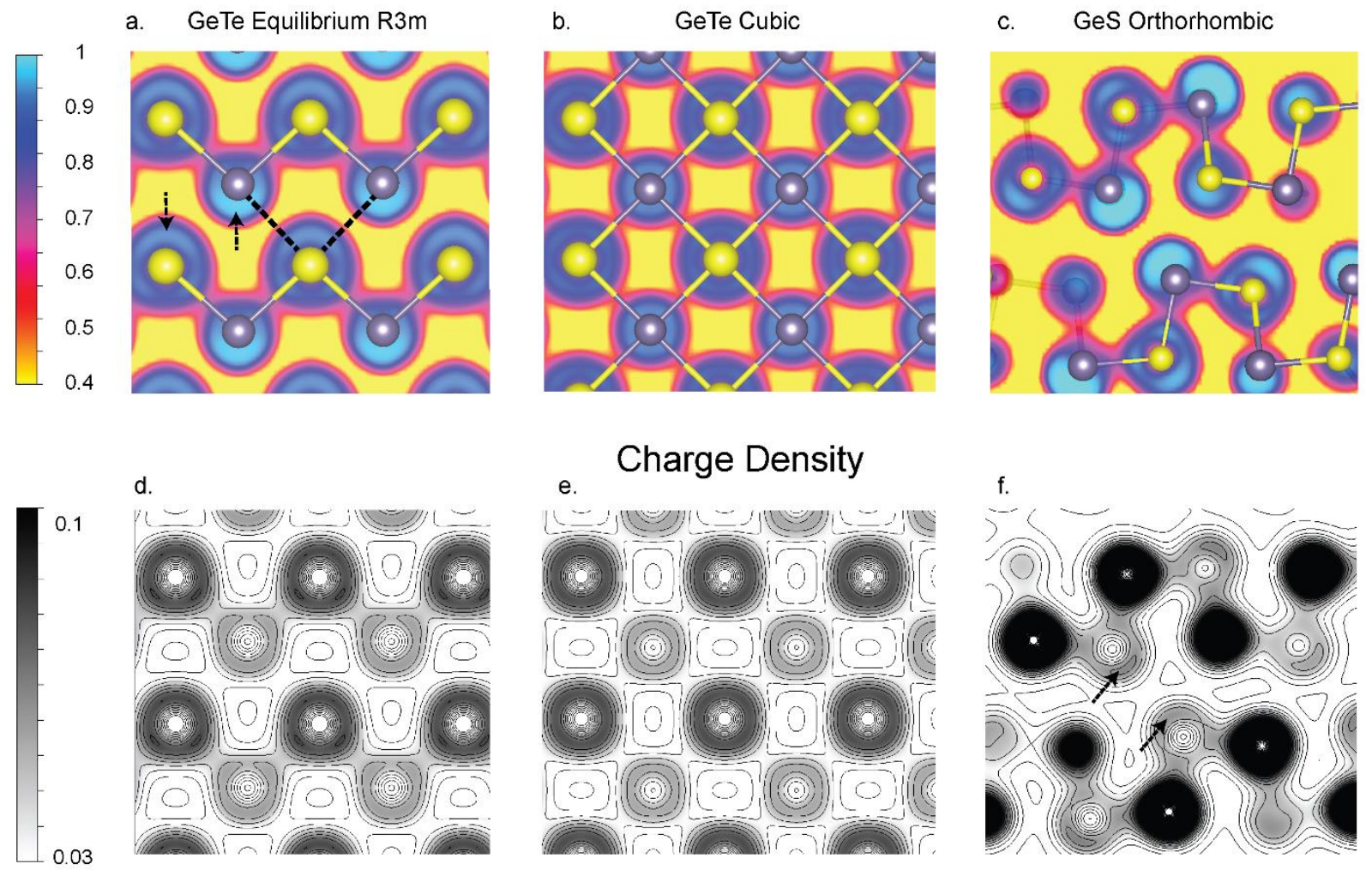

Figure 3: ELF (top panels) and charge density contour plots (lower panels) for GeTe in the equilibrium R-3m structure (a, d), in the relaxed rocksalt structure (b, e) and for GeS in the equilibrium Pnma structure (c, f). The R-3m projection direction corresponds to the $\langle 100\rangle$ direction of the parent rocksalt structure. Note that not all atoms are in plane for the R-3m and particularly for the Pnma structures. In the latter case, the section plane was oriented in order to be as close as possible to the atoms in the center of the image. The contour lines are drawn at 0.01 intervals. Ge atoms are drawn in purple, Te and $\mathrm{S}$ atoms are drawn in yellow. Longer bonds are indicated by dashed lines in GeTe R-3m, and lone pair pockets by arrows in GeS. PBE functional is used.

the bilayers. The angles between first neighbors ranges between 92 and $97^{\circ}$ (as compared to $90^{\circ}$ for the rocksalt phase), whereas the angle between in-plane 'covalent' bonds and interlayer 'van der Waals' bonds $\left(137^{\circ}\right.$ and $\left.148^{\circ}\right)$ is far from the $180^{\circ}$ angle of the original cubic symmetry.

These differences in atomic arrangement are closely related to differences in the density of electronic states (DOS). As can be seen from figure 2, the DOS in the vicinity of the Fermi energy is dominated by p-electrons of both Ge and Te, while s-electrons only play a very minor role (see also Ref. [38]. Hence, bonding is governed by these p-electrons. Upon the transition from the rocksalt structure to the rhombohedral R$3 \mathrm{~m}$ phase the gap in the DOS opens further. Interestingly, this increase the gap (large arrows in Fig.2). However, within the bilayers' plane, the Peierls distortion mechanism is effective (some alignment remains, with a Peierls distortion ratio (PDR) of the short and long bond lengths equal to 1.37), which causes the smaller gap (smaller arrows in Fig.2). In GeS, on the contrary, creating a Peierls distortion in the rocksalt structure has little effect, whereas the gap is larger in the orthorhombic phase.

As mentioned in Refs. [15, 39], the key quantity to understand the structure of IV-VI compounds and compare them with compounds with well-known bonding types (covalent, metallic, ionic) is the degree of localization of the electrons. To this end, ab initio calculations have been 
frequently used to compute the electron localization function (ELF). This function of the electronic density and of the kinetic energy density provides useful information on the degree of 'mobility' of electrons belonging to a region of space, by comparing the kinetic energy to that of the homogeneous electron gas at the same density. In the case of GeTe, the evolution of ELF isosurfaces as a function of the Peierls distortion ratio is shown in Figure $3(a, b)$. An ELF value of 1 indicates perfect electronic localization, where as a value of 0.5 indicates perfect delocalization.

One clearly sees the lower ELF values of the electronic density on the shorter bonds, indicative for a more metallic bond. Yet, the ELF value between layers has to be considered with caution, as the ELF gives no information about the actual density of electrons, only about their kinetic energy. Upon modifying the Peierls distortion, one can only deduce from the ELF that a reduction in distortion ratio is bringing the ELF value close to 0.5 on the longer bonds. Yet, there are also regions where the ELF values are below 0.5 , which can hardly be interpreted.

There is another difficulty linked to the representation of the ELF in Figure 3, which represents the ELF in a selected plane that is defined by two short bonds. Therefore, the electrons involved in the third short bond, which is out of plane can create small pockets. Indeed, in Figure $3 \mathrm{a}$, one could guess the presence of some rather localized electron pockets next to $\mathrm{Ge}$ and Te atoms and located between bilayers (indicated by the arrows). The present ELFs reproduce those published in Ref. [38], however, the analysis of the charge density (Fig. 3d) show that the integrated number of electrons in these pockets is really negligible in GeTe which makes the interpretation in terms of lone pairs (as in Ref. [37]) difficult. It is clearly not the case in orthorhombic $\mathrm{GeS}$ in which lone pairs are clearly seen (Fig. 3f) in the density and correspond to high ELF values (Fig. 3c).

It is actually more interesting to discuss the reduced kinetic energy density along short and longer bonds as shown in Figure 4. Upon going from negative to positive pressure, thus reducing the amplitude of the Peierls distortion, the kinetic energy of the electrons in the long bonds progressively decreases towards the value measured in the center of the shorter bonds. The evolution appears as very continuous. The lowering of the kinetic energy is the clear sign of the progressive delocalization of the electrons upon reducing the Peierls distortion. Inversely, the Peierls distortion effect is to localize the electrons that are delocalized in the symmetric rocksalt structure. The comparison with the orthorhombic structure is also interesting. First, the shorter bonds are similar from the point of view of the kinetic energy between R-3m and Pnma structures. Second, on the longer bonds, the kinetic energy is much lower in the R-3m structure than in the Pnma structure, particularly between the bilayers, indicating a stronger electron delocalization.

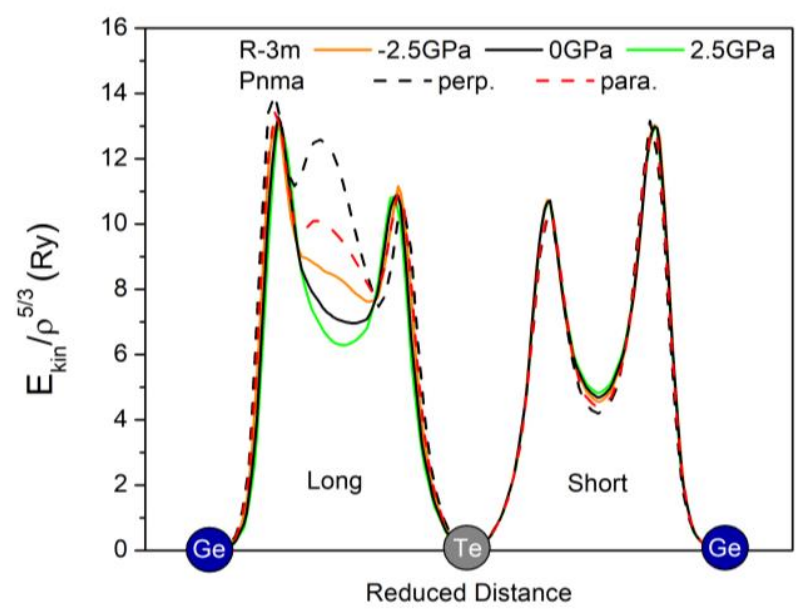

Figure 4: Reduced kinetic energy along Ge-Te bonds computed for relaxed structures and the PBEsol functional. The kinetic energy density value is scaled to the homogeneous electron gas kinetic energy density.

Within DFT, the degree of electronic localization can also be estimated band-by-band using the localization tensor [62], which can be related to the spread of a set of Maximally Localized Wannier Functions (MLWF)[54]. The highest energy valence Wannier functions for GeTe are shown in Figure 5. They constitute a set of localized orbitals obtained by the transformation of the valence band structure from reciprocal to direct space. The diagonal terms of the MLWF Hamiltonians are very close in energy (within $0.5 \mathrm{eV}$ ), the three highest energy MLWF appear as a sigma-type bond on the short Ge-Te distances, one MLWF looks more as an $\mathrm{s}$ orbital, centered on Ge, but extending more towards the interlayer space. The last, lowest energy MLWF is p-like and centered on Te with a main lobe pointing between the bilayers. It cannot be considered as an electronic lone pair for several reasons: the intra layer contribution, the large extension between layers with 3 lobes pointing in the direction of the long interlayer bonds. A detailed inspection of the Slater Hamiltonian shows large resonances between this orbital and the p-sigma ones (about $-2 \mathrm{eV}$ ) indicating the strong hybridization of those orbitals in the valence bands. 
a.

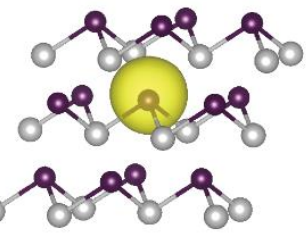

C.

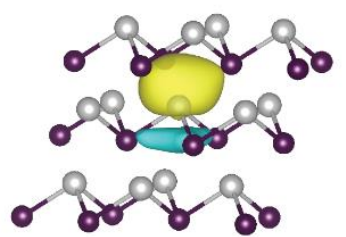

b.

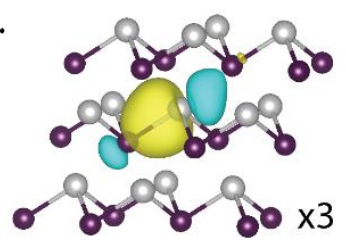

d.

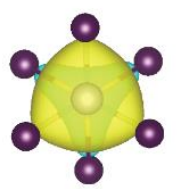

Figure 5 : Wannier functions in GeTe (PBEsol functional). The isosurfaces are drawn at $10 \%$ of the maximal value of the Wannier function in(a). Ge atoms in dark, Te atoms in light color.(a) s-like orbital on $\mathrm{Ge}$, (b) p-sigma on short Ge-Te bond, (c) p-like function centered on Te, seen along the $\langle 111\rangle$ direction in (d).

If one wants to disentangle the various contributions to bonding in GeTe and other IV-VI compounds (covalent, metallic, ionic, van der Waals bonding), it was shown recently that it is necessary to go beyond the one-electron DFT level of theory, as done frequently in quantum chemistry [63]. Recent developments made it possible to extend the calculation of quantum mechanical localization and delocalization bonding indicators to solids and plane-waves orbital basis sets. This has been implemented in the DGRID [64] and Critic2 [57] code, in the latter case with a transformation of the electronic states into MLWF.

The computation of the localization and delocalization indices involves the 2-electron density $\rho\left(\boldsymbol{r}_{\mathbf{1}}, \boldsymbol{r}_{\mathbf{2}}\right)$, which can be expressed as

$$
\rho\left(\boldsymbol{r}_{\mathbf{1}}, \boldsymbol{r}_{\mathbf{2}}\right)=\frac{1}{2}\left[\rho\left(\boldsymbol{r}_{\mathbf{1}}\right) \rho\left(\boldsymbol{r}_{\mathbf{2}}\right)-\rho_{2, x c}\left(\boldsymbol{r}_{\mathbf{1}}, \boldsymbol{r}_{\mathbf{2}}\right)\right]
$$

Where $\rho_{2, x c}\left(\boldsymbol{r}_{\mathbf{1}}, \boldsymbol{r}_{\mathbf{2}}\right)$ is the exchange correlation density, which measures how much an electron is excluded at position $\boldsymbol{r}_{2}$ due to the presence of electron at position $\boldsymbol{r}_{\mathbf{1}}$. It thus includes all non-classical effects. The two-electron density can be computed as the integral of the many-body electron wave function (sometimes in a monodeterminant slater representation, as with the present implementation) over all but two electrons.

If one partitions space into atomic basins, defined here according to Bader's scheme [56], the integration of $\rho_{2, x c}\left(\boldsymbol{r}_{\mathbf{1}}, \boldsymbol{r}_{\mathbf{2}}\right)$ over specific basins yields quantities that have a physical meaning: if $\boldsymbol{r}_{\mathbf{1}}$ and $\boldsymbol{r}_{\mathbf{2}}$ belong to the same atomic basin, the integral yields to the number of electron pairs that are fully localized in that basin, whereas if $\boldsymbol{r}_{\mathbf{1}}$ and $\boldsymbol{r}_{\mathbf{2}}$ are integrated over basins from neighboring atoms, it provides the number of electron pairs that are effectively shared (in a covalent fashion) between those neighbors.

In the case of the ground state rhombohedral structure of GeTe, one finds that 1.24 electrons are shared between neighbors on the shorter bonds, but also that 0.53 electrons (this work, critic 2 calculations in agreement with the DGRID calculations in Ref. [39]) are shared on the long bonds. The long bonds are thus 'true bonds', which explains why the interlayer separation is much smaller than what can be anticipated from the sum of $\mathrm{Ge}$ and Te van der Waals radii. These long bonds are thus not due to lone pairs, which would not appear as pairs of shared electrons, but as a localized pair of electrons instead.

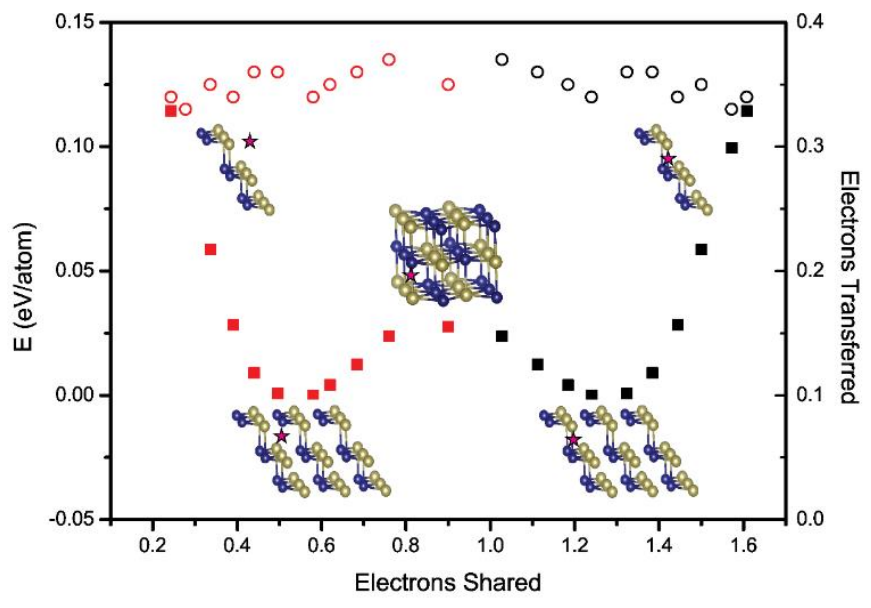

Figure 6: Cohesive energy (filled squares, left axis; the equilibrium $\mathrm{r} 3 \mathrm{~m}$ structure energy is the reference) and the number of transferred electrons (empty symbols, right axis) as a function of the number of electrons shared obtained from the pair density analysis. Values for the shorter bonds are shown in black and longer bonds in red. The insets show representative structures, from quasi-isolated bilayer to equilibrium R-3m and rocksalt. The stars indicate the 'bonds' concerned in each case. Calculations with the GGA PBE functional.

It is also very interesting to note that if one enlarges the Peierls distortion so much that that Ge-Te bilayers become effectively disjoint (see Figure 6), the electrons on the long bonds appear to be fully transferred to the short bonds (which become similar to regular covalent bonds as in Si [39]), but the charge of the atoms is barely changing. It was shown in a recent study of GeTe/SnTe amorphous systems [65] that charge transfer is affecting the features of the Peierls 
distortion. Our results show that ionic bonding plays no major role in the bonding of pure crystalline GeTe.
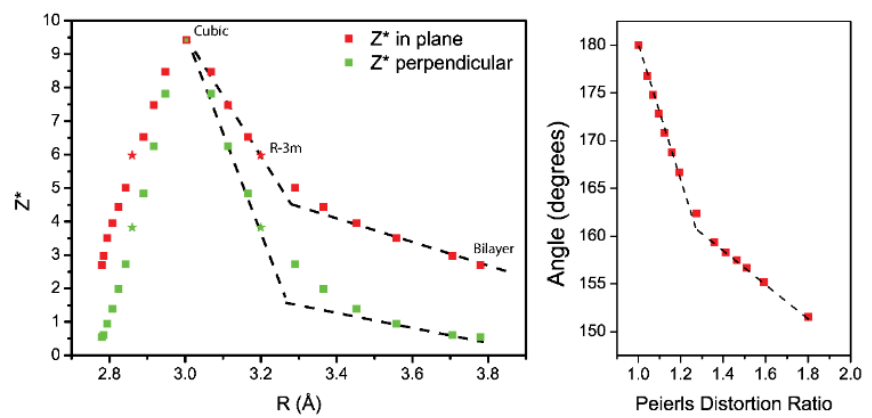

Figure 7. Left: Effective charge tensor values as a function of distance in rhombohedral GeTe with variable distortion ratios (GGA $\mathrm{PBE})$. For each structure relaxed at fixed PDR, 2 distances are obtained. $Z^{*}$ values being atomic quantities, we thus have the same $Z^{*}$ value plotted for two distances. The $Z^{*}$ tensor can then be decomposed into in-plane (red) components and out-of-plane (green) components. At equilibrium, $\left|\mathrm{Z}^{*}\right|$ in plane equals $5.98 \mathrm{e}$ in-plane and 3.82e in-plane, indicated by the stars. Right: The corresponding evolution of the angle between short and long bonds as a function of the Peierls distortion ratio. The dashed lines are guides for the eyes.

Figure 6 also shows that in the cubic limit, about 1 electron $(0.9)$ is contributing to each bond whereas a regular covalent bond would count 2 electrons (actually slightly less than when computed with the pair density analysis due to exchange) . With such a band filling, all structures should be metallic which is not the case here due to Peierls distortion (and some limited charge transfer) in non-cubic cases and charge transfer only in the cubic cases. A natural consequence from this effective half filling is that bonds are 'softer', with low energy transverse optical modes, as shown in ref. [11]. This has a consequence on the thermoelectric figure of merit, which is enhanced $[35,66,67]$. On the other hand, since these bonds that are depleted in electrons in comparison with classical covalently bonded crystals such as silicon, but with more electron sharing than in metals, in which electrons are delocalized over many neighbors [39], they are more polarizable. This is shown in Figure 7. The bond polarizabilities, quantified by the Born effective charge, increase with the reduction of the Peierls distortion, both in the bilayers and across the bilayers. However, this evolution is highly nonlinear, especially in the direction perpendicular to the GeTe bilayers. A clear change occurs when the long bonds gets typically smaller than $3.35 \AA$, which corresponds to a short/long distortion ratio of about 1.19. In the Figure 7 right panel, one can see that the evolution of the angle between shorter and longer bonds also reveals a change of slope around this critical value.

\begin{tabular}{|l|l|l|l|l|l|l|l|l|l|}
\hline & PbTe & SnTe & GeTe & Sb & SnSe & As & SnS & GeSe & GeS \\
\hline PDR & 1 & 1 & 1,12 & 1,17 & 1,2 & 1,25 & 1,26 & 1,32 & 1,38 \\
& & & & & 1,32 & & 1,31 & 1,36 & 1,39 \\
\hline
\end{tabular}

Table 1: Peierls distortion ratio (PDR) for equilibrium crystal structure of IV-VI compounds. For orthorhombic crystals, several distortion ratios are given, according to the directions. The classification of bonding is taken from ref. [39] Values for compounds qualified as metavalently bonded (MVB) are given in italics.

Actually, this ratio seems to be rather universal as it separates metavalent compounds from more ordinary $\mathrm{p}$ bonded IV-VI compounds. The relative contributions of delocalization and Peierls distortion plus charge transfer (localization) to the cohesive energy are shown in Figure 8 where the energy is given for $\mathrm{PbTe}, \mathrm{GeTe}$ and $\mathrm{GeS}$ as a function of the Peierls distortion. The largest distortion values correspond to effectively isolated bilayers.

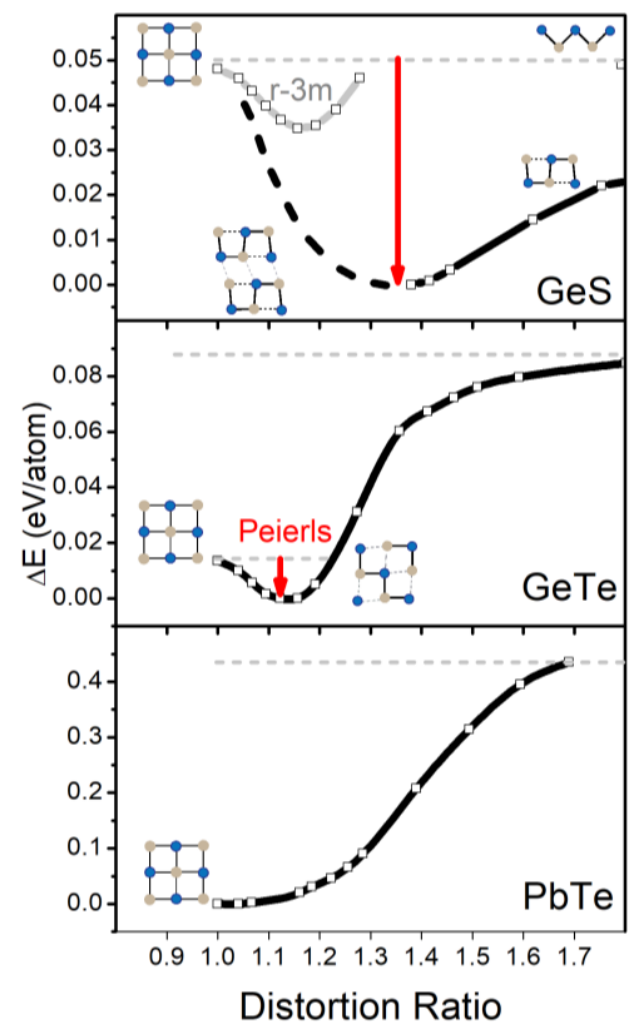

Figure 8: Contribution of the Peierls distortion to the cohesive energy (GGA-PBE functional) in PbTe, GeTe and GeS crystals. The cohesive energy is plotted versus Peierls distortion ratio (see text) with the thick black line. In the GeS orthorhombic crystal, it is not possible to relax structures at fixed PD ratios, and the extrapolated curve is shown as a dashed line. The reference for GeS cubic and covalent limits are obtained in the R-3m phase (grey line).

For $\mathrm{PbTe}$, creating a distortion from the stable cubic structure is ineffective, and most of the cohesive energy results from two combined effects: charge transfer (see Ref. [68] for a comparison with other lead chalcogenides), which prevents 
the compound structure from following the octet rule with 4 fold bonded $\mathrm{Pb}$ atoms and 2-fold bonded Te atoms, and the delocalization of electrons in sigma $\mathrm{p}$-bonds. The metavalent properties appear thanks to the combination of the electron delocalization and the localization arising from charge transfer, that opens the ionic gap. In GeTe, it is the Peierls distortion that is responsible for the creation of the gap with a small stabilization effect $(0.013 \mathrm{eV})$. Still most of the energy comes from the electron delocalization, (a small localization contribution coming from charge transfer is impossible to disentangle at this point). GeTe is metavalent. In GeS, obtaining the path from Pnma to $\mathrm{NaCl}$ structure proves difficult. Also, in isolated bilayers from the orthorhombic phase, the in-plane Peierls distortion remains, which makes it impossible to separate the various contributions to the cohesive energy. Therefore, we computed the R-3m structure as a function of the distortion. It appears clearly forcing the electrons delocalization is not stabilizing the system whereas the Peierls distortion and the larger charge transfer are really effective. GeS is therefore (iono) covalent and not metavalent.

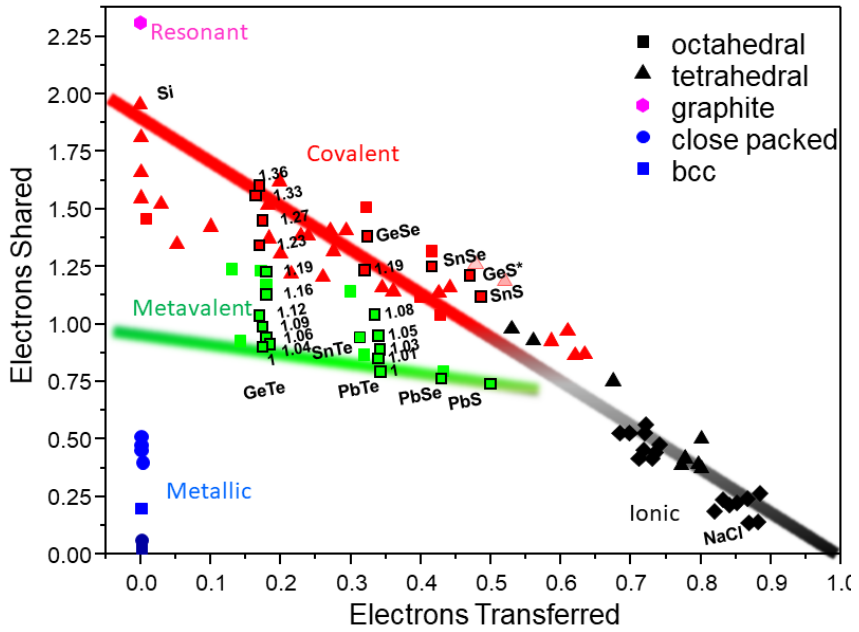

Figure 9: location of IV-VI compounds in an electrons shared (ES) vs electrons transferred (ET) map. Data adapted from Refs. [39,69], except for GeS (this work). Covalent, metallic and ionic dominant character are indicated in red, blue and black, respectively. The green area corresponds to metavalent compounds (definition based on properties as well as on electron sharing/ transfer values, see Refs. $[39,41]$. The red to black line corresponds to a transition from fully covalent to fully ionic bonding, while the green line corresponds to undistorted, cubic compounds with metavalent bonding. Series of points are given for rhombohedral $\mathrm{GeTe}$ and $\mathrm{PbTe}$ with varying Peierls distortion ratios (all cell parameters are relaxed with atomic fractional coordinates fixed).

\section{Conclusion}

Bonding in IV-VI compounds is revisited using DFT and pair-density calculations (see Figure 9). The newly described metavalent bonds are considered and we show that this bonding mechanism is characterized by vanishing or small
Peierls distortions and/or vanishing or small charge transfer. This competition between delocalization and localization creates highly polarizable bonds. The stabilization by MVB occurs by lowering the kinetic energy of the electrons to the contrary of the Peierls distortion or charge transfer (ionic bonding) (also see Ref. [14]). We also show that ionic bonding plays no major role in these compounds and do not evidence any lone pairs.

It should be noted that including Van der Waals interactions, as parametrized on some semi-empirical functionals [70, 71] frequently used to describe IV-VI chalcogenides, is not well suited to the case of MVB systems, as these functionals were fitted to some lone pair systems, and as the anomalous bond polarizability is clearly a characteristics of metavalent bonding.

As we have shown in this, metavalent bonding appears to be a unique fundamental mechanism that differs from the classically described bonding types. It is neither covalent, nor metallic bonding. The bonds are effectively close to halffilled, which explains the anomalies observed experimentally and theoretically, i.e. softness of TO vibrational modes, high polarizabilities and dielectric constants and different bond breaking mechanism. High values of the Peierls distortion or significant charge transfer destroy metavalent bonding. The concept of resonance bonding which has previously been employed to explain the unique properties of materials like $\mathrm{GeTe}$ or $\mathrm{Sb} 2 \mathrm{Te} 3$ appears to be improper. Taking graphene as a prototype of a resonantly bonded compound we see that not only the electron distribution differs from MVB compounds, with localized electrons in covalent $\mathrm{sp} 2$ bonds, whereas in MVB all electrons play the same role and are partly delocalized as shown by the pair density and kinetic energy calculations. This has been very recently evidenced experimentally, with bond breaking in graphite being similar to covalent bonding, whereas all known MVB compounds show an unconventional, collective bond breaking [41, 42].

\section{Acknowledgements}

J.-Y.R. acknowledge the collaborative convention between CEA-LETI and FRS-FNRS. M.W. acknowledges support by the DFG (SFB 917). J.-Y.R. acknowledges computational resources provided by the CECI funded by the F.R.S.-FNRS under Grant No. 2.5020.11 and the Tier-1 supercomputer of the Fédération Wallonie-Bruxelles, infrastructure funded by the Walloon Region under grant agreement $n^{\circ} 1117545$ and support from the ARC AIMED.

\section{References}

[1] Lucovsky G. and White R.M. 1973 Phys. Rev. B 8660

[2] Zachariasen H.W. 1932 J. Am. Chem. Soc. 543841

[3] Wuttig M. and Yamada N. 2007 Nat. Mater. 6824 
[4] http://www.techinsights.com/abouttechinsights/overview/blog/intel-3D-xpoint-memory-dieremoved-from-intel-optane-pcm/

[5] Bruns G., Merkelbach P., Schlockermann C., Salinga M., Wuttig M., Happ T.D., Philipp J.B. \& Kund M. 2009. App. Phys. Lett. 95, 043108

[6] Zhang, W., Mazzarello, R., Wuttig, M., \& Ma, E. 2019. Nat. Rev. Mater. 4, 150

[7] Pries, J., Cojocaru-Mirédin, O., \& Wuttig, M. 2019. MRS Bull. 44, 699

[8] Orava, J., Greer, A. L., Gholipour, B., Hewak, D. W., \& Smith, C. E. 2012. Nat. Mater. 11, 279

[9] Hafermann M, Schöppe P., Rensberg J. and Ronning C 2018 ACS Photonics 55103

[10] Chen W.T., Zhu A.Y., Sisler J, Bharwani Z and Capasso F. 2019 Nat. Commun. 10, 355

[11] Gholipour B., Piccinotti D., Karvounis A., MacDonald K.F. and Zheludev N.I. 2019 Nano Lett. 191643

[12] Cheng Z., Ríos C., Youngblood N., Wright C.D., Pernice W.H.P and Bhaskaran H. 2018 Adv. Mater. 301870238

[13] Lee T.H. and Elliott S.R. 2019 arXiv 1909.05281.

[14] Jones R. 2018 J. Phys. Condens. Matter 30153001

[15] Wuttig M., Deringer V.L, Gonze X., Bichara C. and Raty J.Y. 2018 Adv. Mater. 301803777

[16] Mukhopadhyay S., Sun J., Subedi A., Siegrist T. and Singh D.J.2016 Sci. Rep. 625981

[17] Raty J.Y., Zhang W., Luckas J., Chen C., Mazzarello R., Bichara C. and Wuttig M 2015 Nat. Commun. 67467

[18] Littlewood P.B. 1980 J. Phys. C Solid State Phys. 134855

[19] Littlewood P.B. 1980 J. Phys. C Solid State Phys. 134875

[20] Dutta S.N. and Jeffrey G.A. 1965 lnorg. Chem. 41363

[21] Cohen M.H., Falicov L.M. and Golin S. 1964 IBM J. Res. Dev. 8215

[22] Gaspard J.P., Marinelli F. and Pellegatti A. 1987 EPL 31095

[23] Gaspard J.P., Pellegatti A., Marinelli F. and Bichara C. 1998 Phil. Mag. B 77727

[24] Chattopadhyay T., Boucherle J.X.and von Schnering H.G. 1987 J. Phys. C Solid State Phys. 201431

[25] Chattopadhyay T., Werner A., von Schnering H.G and Pannetier J. 1984 , Applied Phys. 19807

[26] Raty J.Y., Otjacques C., Gaspard J.P. and Bichara C. 2010 Solid State Sci. 12193

[27] Raty J.Y., Godlevsky V., Ghosez P., Bichara C., Gaspard J.P. and Chelikowsky J.R. 2000 Phys. Rev. Lett. 851950

[28] Raty J.Y., Otjacques C., Peköz R., Lordi V. and Bichara C. 2015 in Molecular Dynamics Simulations of Disordered Materials: From Network Glasses to Phase-Change Memory Alloys (Eds: C. Massobrio, J. Du, M. Bernasconi and P. S. Salmon), Springer, Cham, Switzerland 485

[29] Raty J.Y., Godlevsky V., Gaspard J.P., Bichara C., Bionducci M., Bellissent R., Céolin R., Chelikowsky J.R. and Ghosez P. 2001 Phys. Rev. B 64235209

[30] Shportko K., Kremers S., Woda M., Lencer D., Robertson J. and Wuttig M. 2008 Nat. Mater. 7653

[31] Matsunaga T., Yamada N., Kojima R., Shamoto S., Sato M., Tanida H., Uruga T., Kohara S., Takata M., Zalden P., Bruns G., Sergueev I., Wille H.C., Hermann R.P. and Wuttig M. 2011 Adv. Funct. Mater. 212232
[32] Kolobov A.V., Fons P., Frenkel A.I., Ankudinov A.L., Tominaga J. and Uruga T. 2004 Nat. Mater. 3703

[33] Lencer D., Salinga M., Grabowski G., Hickel H., Neugebauer J. and Wuttig M 2008 Nat. Mater. 7972

[34] Huang B. and Robertson J. 2010 Phys. Rev. B 81081204

[35] Lee S., Esfarjani K., Luo T., Zhou J., Tian Z. and Chen G. 2014 Nat. Commun. 53525

[36] Kolobov, A. V., Fons, P., \& Tominaga, J. 2013. Phys. Rev. B, 87, 155204

[37] Kolobov, A. V., Fons, P., \& Tominaga, J. 2012. Phys. status solidi (b), 249, 1902

[38] Waghmare, U. V., Spaldin, N. A., Kandpal, H. C., \& Seshadri, R. 2003. Phys. Rev. B, 67, 125111

[39] Raty J.Y., Schumacher M., Golub P., Deringer V.L., Gatti C. and Wuttig M. 2019 Adv. Mater. 311806280

[40] Cheng Y., Cojocaru-Mirédin O., Keutgen J., Yu Y., Küpers M., Schumacher M., Golub P., Raty J.Y., Dronskowski R. and Wuttig M. 2019 Adv. Mater. 311904316

[41] Zhu M., Cojocaru-Mirédin O., Mio A.M., Keutgen J., Küpers M., Yu Y., Cho J.Y., Dronskowski R. and Wuttig M 2018 Adv. Mater. 301706735

[42] Raghuwanshi M., Cojocaru-Mirédin O. and Wuttig M 2020 Nano Lett. 20116

[43] Gonze X., Beuken J.M., Caracas R., Detraux F., Fuchs M., Rignanese G.M., Sindic L., Verstraete M., Zerah G., Jollet F., Torrent M., Roy A., Mikami M., Ghosez P., Raty J.Y. and Allan D.C. 2002 Comput. Mater. Sci. 25478

[44] Kresse G. and Hafner J. 1993 Phys. Rev. B 47558

[45] Kresse G. and Furthmuller J. 1996 Phys. Rev. B 5411169

[46] Giannozzi P., Baroni S., Bonini N., Calandra M., Car R., Cavazzoni C., Ceresoli D., Chiarotti G.L., Cococcioni M, Dabo I., Dal Corso A., de Gironcoli S., Fabris S., Fratesi G., Gebauer R., Gerstmann U., Gougoussis C., Kokalj A., Lazzeri M., Martin-Samos L., Marzari M., Mauri F., Mazzarello R., Paolini S., Pasquarello A., Paulatto L., Sbraccia C., Scandolo S., Sclauzero G., Seitsonen A.G., Smogunov A., Umari P. and Wentzcovitch R.M. 2009 J. Phys. Condens. Matter 21395502

[47] Hamann D.R. 2013 Phys. Rev. B 88085117

[48] Hartwigsen C., Goedecker S. and Hutter J. 1998 Phys. Rev. B $\mathbf{5 8 , 3 6 4 1}$

[49] Perdew J.P., Burke K. and Ernzerhof M. 1996 Phys. Rev. Lett. 773865

[50] Perdew J.P., Ruzsinszky A., Csonka G.I., Vydrov O.A., Scuseria G.E., Constantin L.A., Zhou X. and Burke K. 2008 Phys. Rev. Lett. 100136406

[51] Ibarra-Hernández, W., \& Raty, J. Y. 2018 Phys. Rev. B 97, 245205

[52] Gonze X., Allan D.C. and Teter M.P. 1992 Phys. Rev. Lett. 68 3603

[53] Golub P. and Baranov A.I. 2016 J. Chem. Phys. 145154107

[54] Marzari N. and Vanderbilt D. 1997 Phys. Rev. B 5612847

[55] Mostofi A.A., Yates J.R., Lee Y.-S., Souza I., Vanderbilt D. and Marzari N. 2008 Comput. Phys. Commun. 178685

[56] Yu M. and Trinkle D.R. 2011 J. Chem. Phys. 13406411

[57] Otero-de-la-Roza A., Johnson E.R. and Luaña V. 2014 Comput. Phys. Commun 1851007

[58] Shaltaf R., Durgun E., Raty J.Y., Ghosez P. and Gonze X. 2008 Phys. Rev. B 78205203.

[59] Rabe K.M. and Joannopoulos J.D. 1987 Phys. Rev. B 363319 
[60] Edwards A.H., Pineda A.C., Schultz P.A., Martin M.G., Thompson A.P. and Hjalmarson H.P. 2005 J. Phys. Condens. Matter 17 L329

[61] Maintz S., Deringer V.L., Tchougréeff A.L. and Dronskowski R. 2016 J. Comput. Chem. 371030

[62] Veithen M., Gonze X. and Ghosez P. 2002 Phys. Rev. B 66 235113

[63] Gatti C. and Macchi P. 2011 in Modern Charge-Density Analysis (Eds: Springer), pp. 1-78.

[64] Kohout M. 2019 DGrid 5.1 Dresden

[65] Chen, Y., Sun, L., Zhou, Y., Zewdie, G. M., Deringer, V. L., Mazzarello, R., \& Zhang, W. 2020. J. Mater. Chem. C 8, 71

[66] Yu Y., Cagnoni M., Cojocaru-Mirédin O. and Wuttig M 2019 Advanced Functional Materials 1904862

[67] Cagnoni M., Führen D. and Wuttig M. 2018 Adv. Mater. 30 1801787

[68] Maier S., Steinberg S., Schumacher M., Golub P., Raty J.Y., Nelson R., Mazzarello R., Conjocaru-Mirédin O., Dronskowski R. and Wuttig M. 2020 to be submitted

[69] Kooi B.J. and Wuttig M. 2020 Adv. Mater. under review.

[70] Grimme S. 2006 J. Comput. Chem. 271787

[71] Grimme S., Antony J., Ehrlich S. and Krieg H., J. Chem. Phys. 132154104 